Voix et Images

voixetimages

\title{
Louis Jolliet vu par Alain Grandbois ou l'Histoire au service du mythe
}

\section{François Gallays}

Volume 5, numéro 1, automne 1979

Jacques Godbout

URI : https://id.erudit.org/iderudit/200189ar

DOI : https://doi.org/10.7202/200189ar

Aller au sommaire du numéro

Éditeur(s)

Les Presses de l'Université du Québec

ISSN

0318-9201 (imprimé)

1705-933X (numérique)

Découvrir la revue

Citer cet article

Gallays, F. (1979). Louis Jolliet vu par Alain Grandbois ou l'Histoire au service du mythe. Voix et Images, 5(1), 65-86. https://doi.org/10.7202/200189ar d'utilisation que vous pouvez consulter en ligne.

https://apropos.erudit.org/fr/usagers/politique-dutilisation/ 


\title{
Louis Jolliet vu par Alain Grandbois ou l'Histoire au service du mythe
}

Premier livre d'Alain Grandbois, publié d'abord à Paris en 1933 et repris à Montréal chez Fides en 1948, Né à Québec 1 fut qualifié par l'auteur de récit. Sur la couverture, entre le titre et sa caractérisation générique, l'auteur y a inscrit en sous-titre le nom du découvreur du Mississippi : Louis Jolliet. Ainsi, dès avant la lecture de la première ligne du texte, le lecteur sait qu'il se trouve devant une cuuvre en quelque sorte hybride, puisqu'il s'y mêlera deux discours: celui de l'histoire, c'est-à-dire du réel reconstitué et celui de l'imaginaire. Que ce dernier participera massivement à l'élaboration de l'œuvre, très tôt les signes se multipliant le diront. Outre la caractérisation du titre, la dédicace:

\author{
A MON PERE \\ qui enchanta mon enfance \\ en me racontant de belles \\ histoires
}

non seulement nomme le dédicataire, mais contient aussi sa propre justification. De plus, ce faisant, Grandbois, peut-être à son insu, livre à ses lecteurs éventuels l'origine de son récit: ces «belles histoires" racontées par son père. Aussi, on peut le deviner, l'Histoire lui fut d'abord présentée comme une série d'histoires, le déroulement d'une suite ininterrompue de récits mettant en scène des protagonistes, hommes et femmes, hissés au rang de héros et héroïnes, dont les qualités étaient telles qu'ils furent des exemples à vouloir imiter (ce furent de abelles histoires"). Outre cela, toujours dans la dédicace, le verbe qu'emploie Grandbois pour exprimer l'effet qu'eurent ces histoires sur son esprit indique assez clairement que celles-ci contribuèrent largement à la formation de son imaginaire. Toujours en s'appuyant sur la dédicace on pourrait même ajouter que chez Grandbois les fantasmes de son enfance ont pendant longtemps présidé à ses activités. On dirait que, parvenu à l'âge mûr, Grandbois a voulu d'une certaine manière se substituer à son père: plutôt que de subir l'enchantement il a cherché, à son tour, à en produire. L'on touche ici, il me semble, au projet que s'est donné Grandbois dans Né à Québec: par la relation d'une «belle histoire", la production de 
l'enchantement. Que Né à Québec fut le premier texte à naître sous la plume de Grandbois me paraît significatif: il est le lieu inscriptif d'une sorte de passage rituel - et, simultanément, le résultat - de la passivité réceptive à l'activité créatrice. II marque la volonté de donner corps à ses fantasmes, de leur donner l'épaisseur du réel, de ne plus se contenter, en d'autres mots, de simplement les rêver.

Donner corps à ses fantasmes... N'est-ce pas ce que fait Grandbois dès la première page de son récit lorsque, pour motiver l'émigration d'Adrien d'Abancourt ${ }^{2}$, grand-père maternel de Louis Jolliet, vers la Nouvelle France, il prête à ce personnage une imagination fertile nourrie par les lectures des exploits des grands voyageurs ? ${ }^{3}$ Etant donné le mutisme à cet égard des documents historiques, c'est évidemment de toutes pièces que Grandbois a inventé le début de ce récit. Et les rêves qu'il prête au jeune d'Abancourt, ces désirs de voyages, ce goût des contrées lointaines, crés et entretenus par la lecture des récits de voyage, sont, à n'en pas douter, ceux-là même de l'auteur:

Dans la nuit du même jour, il fit clair de lune et un marin, qui s'appelait Juan Rodrigo Bernejo, habitant de Molino, dans la campagne de Séville, et qui faisait partie de l'équipage du navire de martin Alonso Pinzon, aperçut à la clarté de la lune une langue blanche de sable et, levant les yeux, il vit la terre. II tira aussitôt un coup de bombarde en criant : «Terre! Terre! »... Ce passage du voyage de Colomb éblouissait Adrien d'Abancourt, lui paraissait la plus belle chose du monde. [...] Un sortilège opérait. Le jeune garçon quittait les Amériques pour les mondes fabuleux de Jean de Mandeville, de Marco Polo. Son imagination tissait des rêves héroïques. Il se voyait à la proue d'une caravelle, droit et fier, les poings aux hanches, remontant parmi des îles d'or le cours d'un fleuve bleu. Et des princes vêtus de lin blanc, sur des jonques de porcelaine, l'acclamaient et ils lui offraient des palais, des richesses, des armées. Lui passait, dédaigneux, et il s'enfonçait dans les profondeurs des continents. Là, il livrait d'effroyables batailles, il soumettait des empires. Il avançait toujours, nimbé d'une gloire fulgurante. Et son nom rougeoyait comme un incendie... [...] Avec les années, le jeune Abancourt ne perdit point le goût des voyages. II conquit le Mexique avec Cortez, accompagna Pizarre au Pérou, navigua sur le Rio de la Plata aux côtés de Juan Diaz de Solis, subit avec les frères Cabot, de monstrueuses tempêtes océanes, pénétra, avec le navigateur Cartier, les mystères brumeux du golfe nordique conduisant aux Canadas. II apprit ainsi que les hommes rouges nommaient le soleil Ysnay et le feu Azista. Ces syllabes l'enchantèrent (p. 14).

Dans cet extrait, volontairement long, où Grandbois prête à son personnage ses propres rêves, meublés d'aventures et d'actes héroïques, où s'exprime aussi une volonté délirante de puissance, rêves, en somme, d'un adolescent imaginatif, deux verbes retiennent l'attention: ce sont les deux verbes commentatifs qu'emploie l'auteur pour dire, à deux reprises, l'attitude du jeune d'Abancourt. Après le récit, dans les toutes premières lignes du texte, de la découverte d'Amérique par Christophe Colomb, Grandbois pour 
exprimer l'émoi de son personnage devant la relation de cet événement, emploie le verbe éblouir. Et devant les deux mots indiens Ysnay et Azista, Abancourt, dit Grandbois, fut enchanté. Ces mots - qui présentent une certaine synonymie et dont l'un, sous la forme verbale, reprend un des substantifs que contient la dédicace du livre (enchantement) - plutôt que d'être l'expression de l'attitude d'Abancourt dans la réalité, puisque Grandbois n'en savait strictement rien, expriment, me semble-t-il, bien plus les motivations de Grandbois à l'origine de son propre texte. Car qu'est-ce qui provoque l'éblouissement et l'enchantement dont fait état le texte de Grandbois sinon d'autres textes: l'un, grâce à son contenu hautement dramatique: la découverte d'Amérique, l'autre, grâce à sa matérialité même: les sons enchanteurs de mots étrangers? Depuis les textes oraux du père, en passant par les textes écrits, qui de Colomb, qui de Champlain, jusqu'au propre texte de Grandbois, on peut voir comme la naissance et le développement d'un destin ou, plus précisément, d'un double destin: celui du voyageur et celui de l'écrivain/poète. Tout se passe comme si, nourri des exploits des grands découvreurs, à une époque de sa vie où s'effectue chez l'enfant la cristallisation du moi et ce, par l'entremise de ce que lui propose son milieu, Grandbois avait d'abord rêvé son destin. Et Né à Québec ne serait pas autre chose que la relation, par l'entremise de Jolliet, de ses propres désirs. Grâce à la silhouette de ce lointain ancêtre qu'est Jolliet, Grandbois n'a-t-il pas, en la faisant revivre, d'une certaine façon, conjugué dans une seule réalisation les deux volets constitutifs de ce qui sera désormais sa vie: le goût de l'aventure et du voyage et le goût du langage et de l'écriture? Produit d'une double fascination, celle des "belles histoires" et celle du "langage", Né à Québec mérite, je crois, qu'on l'examine de près sous ce double aspect.

"II (Alain Grandbois) a voulu faire œuvre d'historien et, affirme Jacques Blais ${ }^{4}$, pour l'essentiel, tout donne à croire qu'il y soit parvenu. Les dates sont justes; l'itinéraire, exact. Les anecdotes sont bien celles que rapporte Jolliet lui-même dans la lettre, au retour, à Frontenac. " Pourtant quelques lignes plus bas, Blais, bien délicatement, se met à l'abri : "Imitons ici la prudence de l'abbé Groulx selon lequel “si Né à Québec n'est pas à proprement parler un ouvrage d'historien, nul ne refusera d'y reconnaître l'œuvre d'un grand poète." " Que Grandbois se soit documenté sérieusement, on ne peut avoir là-dessus aucun doute: les traces de ses nombreuses lectures se retrouvent à chaque page de son récit. Mais a-t-il pour autant "voulu" faire œuvre d'historien? Selon moi, cette volonté ne se manifeste nullement dans son œuvre. Bien au contraire, en plus de la qualification qu'a donné Grandbois lui-même à son texte - celle de récit - celui-ci contient nombre d'indices révélant une intention qui tendrait à infirmer ce qu'avance Jacques Blais. 
En fait, tout porte à croire que les documents historiques ne furent pour Grandbois que des matériaux bruts dont il s'est servi pour l'élaboration d'une œuvre dont la finalité fut autre qu'historique, d'où sa liberté d'usage à leur égard. Que fut sacrifiée l'exactitude historique, lorsque celle-ci entrait en conflit avec le but poursuivi ne signifie pas, toutefois, que Grandbois n'en ait pas eu le souci. Car, dans la fabrication de son texte, s'il ne voulait pas tomber dans le piège auquel ne sut échapper, par exemple, Constantin Weyer dans son livre Épopée canadienne: Cavelier de La Salle, où les faits historiques sont pour ainsi dire complètement noyés dans la fiction, Grandbois se devait de créer l'impression de respecter les faits. Et c'est sans doute ce qui fonde la valeur de Né à Québec.

L'auteur a réussi à fabriquer un récit, c'est-à-dire un texte de fiction, d'où se dégage, néanmoins, à la lecture, une impression d'authenticité historique. Comment au juste s'y est-il pris ? D'abord et avant tout, il me semble, en intégrant à son propre texte les traces multiples des textes de l'époque: soit par le biais de la citation - extraits de lettres de Talon, de Frontenac; paroles de Marie de l'Incarnation, de Ragueneau, de Champlain, de SaintLusson ou d'un vieux capitaine algonquin - sont par l'inscription de noms d'auteurs de textes: Lejeune, Dablon, Dollier. Ayant emprunté à l'érudit certaines de ses méthodes de construire un texte, Grandbois s'assura par làmême une garantie de sérieux et de semblant de vérité qui ne peut qu'agir très favorablement sur le lecteur. Agit aussi de manière favorable sur le lecteur la datation précise des événements relevés: “... le 29 mars 1632, le traité de Saint-Germain-en-Laye... (p. 28)"; "le 29 octobre 1639, Marie d'Abancourt... (p. 30) »; « Le 14 novembre 1674, Frontenac écrivait à Golbert... (p. 163)". Cependant, à côté de ces dates où tout y est: le jour, le mois, l'année, il se trouve dans le récit quantité de dates où seuls sont retenus le jour et le mois: "Le 20 mars tout était prêt... (p. 46) "; "Le 14 septembre, MM. de Tracy et de Courcelle... (p. 77)"; "Voici l'été. Juin, juillet, août. Le 18 Talon débarqua... (114). "Le 12 du même mois, il fit enregistrer ses lettres... (p. 127)". La liste pourrait se prolonger presque indéfiniment, mais ces exemples suffisent à faire comprendre le procédé: pour aucune de ces dates tronquées, le texte ne permet de restituer l'année de sorte que, telles quelles, pour fin de relier l'événement dont il est question à un cadre temporel référentiel, ces dates ne sont d'aucun secours, bien que - et ceci est capital - elles puissent en donner l'illusion. Et dans ce sens, elles ne font pas autre chose que de créer ce que Barthes a appelé un « effet de réel». Par ailleurs, ce type de précision temporelle assure la fonction de faire progresser le récit. Peu importe si aucune autre date n'a été auparavant précisée, son insertion dans le texte $y$ imprime la marque d'une progression, d'un passage, d'une modification dans le cadre narratif. De plus, le foisonnement de ce type de datation finit par créer un effet de décollement par rapport au réel historique, puisque l'événement historique, proprement insituable quoiqu'illusoirement situé, se met à exister en fonction de ce qui précède et de ce qui suit, c'est-à-dire non plus en fonction du cadre chronologique réel du passé mais en fonction du récit qui se construit. 
A côté de cet usage particulier des dates, s'inscrit aussi l'usage que fait Grandbois de certaines expressions d'ordre temporel. Ces termes, en faisant référence aux saisons de l'année, soulignent le passage du temps et, tout comme l'usage des dates incomplètes, assurent la progression du récit cependant qu'ils empêchent le repérage chronologique exact de l'événement emprunté à l'histoire: "La fin de l'automne n'était pas venue qu'il (M. de Courcelles) résolut brusquement d'aller guerroyer contre les Iroquois... (p. 72)». " Aux premiers jours du printemps... (p. 120) »; « Une nuit, par un froid glacial, un incendie dévora la mission. (p. 120) m. Encore ici, rien dans le texte ne permet de préciser l'année exacte durant laquelle eurent lieu les événements. Mais le meilleur exemple, je crois, de ce genre d'usage est celui-ci: "L'automne vint (p. 66)". Cette phrase laconique, Grandbois l'a employée comme charnière entre la relation de deux faits historiques bien distincts: le premier fait état, en un paragraphe, de la persécution que dut endurer la colonie entre les années 1648 et 1652 aux mains des Iroquois et le second rappelle le " tremble-terre" qui eut lieu le 5 février 1663. Or, cette phrase de transition, sur le plan temporel, établit un lien de succession immédiate entre la situation qui précède et l'événement qui suit. Cependant, on l'a vu, chronologiquement, cela est impossible. Ainsi, en renvoyant aux cycles des saisons, à un temps donc se déroulant sans faille, ce type d'expression permet de masquer l'état lacunaire du temps historique en créant, en quelque sorte, une lisse surface narrative sous laquelle se cache la réalité fragmentaire du passé. De plus, du strict point de vue logique, cette phrase, puisqu'elle est précédée d'une description situationnelle, devrait être suivie d'une action modificatrice de la situation. En d'autres termes, du point de vue discursif, cette phrase annonce une modification à l'intérieur de la même isotopie, alors qu'ici elle fait passer le texte d'une isotopie à une autre.

Tandis que les expressions temporelles fondées sur le cycle des saisons permettent, par voie de consécution, le développement du récit, d'autres expressions, temporelles aussi, le développement dans la simultanéité. Ainsi les expressions telles que: "Pendant ce temps... (p. 44)"; “... Cependant, sur les bords... (p. 52) " servent dans Né à Québec de liaison à des événements qui, historiquement, n'ont pas forcément eu lieu au même moment et, surtout, qui, logiquement, ne jouissent d'aucune relation commune. Un exemple : la messe de minuit, célébrée par Monseigneur Laval à Québec à laquelle assista Monsieur d'Argenson, d'une part, et la méditation des capitaines Iroquois "à la lueur de feux rouges" sur les bords de l'Ontario, d'autre part. C'est là employer des artifices, des "organisateurs de récits", selon Phillippe Hamon ${ }^{5}$, très familiers aux romanciers mais dont se méfie avec raison l'historien. Par ailleurs, ce rapprochement fonctionne avec efficacité au niveau historique du récit. Cela est vrai aussi pour les noms de lieux que contient le texte.

L'historien, lorsque son étude porte sur un état antérieur de civilisation où tout aussi bien les repères géographiques naturels que les villes et villages d'alors furent connus sous des noms différents, I'historien, dis-je, par souci de clarté, assurera, dès le début, la compréhension de l'ancienne 
toponymie par la superposition à celle-ci de la toponymie actuelle. Or dans Né à Québec, ce procédé est à tel point absent qu'il est impossible de ne pas y voir le résultat d'un parti pris de la part de l'auteur. Bien sûr, point n'est besoin de grands pouvoirs de divination pour reconnaitre ce que sont devenus aujourd'hui Chicagou (p. 157), Manatte (p. 102), ou Chigoutimit (p. 172), mais déjà Catarocouy (p. 158) est plus difficilement repérable sans parler de l'île de Manitoubi. Et à première vue, en voyant le nom de Metsa-Sabé (p. 110) celui de Mississippi ne vient pas nécessairement immédiatement à l'esprit. Bien malin aussi le lecteur qui devinera que là où se situait jadis au temps de Jolliet le village indien de Tinnaouatoua se dresse aujourd'hui la ville de Hamilton. Mais il arrive aussi que l'auteur, plutôt que de donner le terme archaïque (procédé courant), omette tout simplement de nommer le lieu dont il est question. Ainsi, parlant du village iroquois de Gonnontoha, l'auteur fait mine seulement de le situer: "Gonnotoha était situé sur les bords d'un lac, au centre d'une région arrosée de sources d'eaux vives (p. 44)". Précision de romancier et non d'historien. Un peu plus loin, Grandbois reprend le même procédé: “Le 28 du même mois, l'armée entière, composée de trois cents hommes, se réunissait au fort Sainte-Anne, bâti au milieu d'un lac, sur une île (p. 77) ». Ailleurs, le nom est tout simplement omis : "Puis on navigua deux jours sur un lac à fond de roc (p. 104)". Or, combien de lecteurs auront deviné qu'il s'agit là du lac Clair?

On le voit, non seulement Grandbois ne vient pas au secours de son lecteur pour l'aider à reconnaître dans la géographie d'aujourd'hui celle d'hier ou inversement, mais il lui enlève parfois cette possibilité. C'est dire que, du point de vue géographique, Grandbois a pratiqué sur son texte un brouillage systématique apte à produire ainsi chez le lecteur un effet déréalisant. Grâce à ce brouillage, la relation entre les toponymes d'hier et ceux d'aujourd'hui se faisant difficilement, ces noms de lieux perdent de leur réalité mais gagnent, par contre, à cause de leur étrangeté (maintenue intacte), une résonnance quasi mythique. La réduction au réel ne s'étant pas faite, ces noms de lieux maintiennent envers le lecteur une disponibilité pour un certain investissement onirique. C'est une entrave de moins, si l'on veut, à la construction de cette géographie imaginaire à laquelle le texte convie le lecteur. Cependant, ces noms de lieux ne contribuent pas moins à créer chez le lecteur l'impression que ce texte, assis sur une érudition solide, respecte scrupuleusement la "vérité historique». Cela vaut aussi pour l'usage que fait l'auteur des noms des personnages historiques qui prennent place dans son récit.

Exception faite de Jolliet, aucun des personnages n'est, à aucun moment, situé par rapport à l'Histoire; il n'est le plus souvent présent dans le récit que pour un seul événement à la suite de quoi il disparaît pour de bon. Mais hors cet événement, quel rôle a-t-il joué dans la vie de la NouvelleFrance? Combien de temps fut-il là ? A quel moment y fut-il et, parfois, en qualité de quoi? Toutes des questions qui surgissent bien normalement dans l'esprit du lecteur mais qui restent sans réponse. Ou alors si le texte fournit quelques renseignements sur un personnage, ils ressortissent plus propre- 
ment au genre romanesque qu'à l'Histoire, fourmillant de détails de type mondain et de sous-entendus libertins :

M. de Frontenac était le filleul de Louis XIII. II avait I'habitude des camps et de la cour. II avait guerroyé dans les Flandres, en Italie, en Allemagne. II possédait à un haut degré l'art du commandement. Il avait passé l'âge des fredaines et atteint celui des ambitions. La comtesse de Frontenac n'avait pas été étrangère à sa nomination. C'était une femme de beaucoup de gråce. Protégée de $M$. de Ludes, grand maître de l'Artillerie, elle avait, à l'Arsenal, des appartements qu'elle partageait avec son amie, Mlle d'Outrelaise. On les appelait les Divines. Les mauvaises langues prétendirent que la comtesse était fort aise que ses relations avec Frontenac dussent se borner, dorénavant, à un échange de lettres (p. 126).

Mais c'est en examinant d'un peu plus près le personnage central du livre que se dévoilent, selon moi, les véritables intentions de l'auteur et qui sont loin d'être, comme on le verra, celles-là même qui président ordinairement à l'élaboration de l'œuvre d'un historien. D'abord, une remarque s'impose d'elle-méme tellement elle est évidente: Grandbois présente toujours son personnage sous un jour extrêmement favorable.

Examinons, premièrement, l'attitude des Indiens à son égard. Auprès d'eux, le discours de Jolliet, tel que le présente l'auteur, c'est-à-dire sage, honnête et franc, fut toujours efficace. Et nous savons, n'est-ce pas, quels bons juges furent les Indiens pour juger de la valeur du discours d'autrui ! Je ne donnerai comme exemple que ce discours prononcé, selon Grandbois, par Jolliet devant la tribu réunie des Keinouchés sur les bords du Lac Supérieur (mais qui, en réalité ne fut jamais prononcé, car ce fut Adrien, son frère aîné, qui fut commissionné en 1676 par Talon pour aller avec Jean Péré à la recherche du cuivre sur les bords du Lac Supérieur $\left.{ }^{6}\right)$. Ce discours fictif que Grandbois prête à Louis Jolliet fut prononcé dans le but de se faire céder un prisonnier Iroquois que détenaient les Keinouchés. Le discours terminé, le chef indien reprend: “Tes paroles ont changé le cours de leur destin. Nous attendrons les événements futurs. Fais ton choix! (p. 95) ". Bien sûr, non exempte d'une certaine solennité appuyée, qui tient du stéréotype, cette réplique souligne néanmois le pouvoir inhérent au discours de Jolliet : celui de changer les destins, un pouvoir, si l'on veut, qui appartient à une instance surhumaine. Et, en effet, si Grandbois ne fait de son héros une divinité, il le situe cependant bien au-delà de la moyenne des hommes, c'est-à-dire dans cet espace mythique qui sépare les dieux du commun des mortels. Bref, dans Né à Québec, Grandbois crée un être de légende, un demi-dieu, et il n'est pas jusqu'à la description de son physique qui n'ait contribué à sa divination: "Talon le regarde. Très grand, large d'épaules, droit comme un jeune arbre. Magnifique tête bouclée. Dans la face hâlée, des yeux couleurs d'eau de mer (p. 115)". C'est la seule description physique de son personnage qu'ait risquée Grandbois et elle semble tout droit sortie des récits légendaires scandinaves. Mais à bien y penser, les yeux bleus et les cheveux blonds bouclés, ne sont-ce pas là les indices signalétiques de tous les jeunes dieux 
légendaires qui, tels des soleils (boucles d'or), dominent du haut de leur splendeur quasi divine le grouillement des hommes? De plus, pour la construction des deux figures (métaphore et comparaison) que contient cette description, Grandbois a emprunté à la nature les deux éléments qui, dans un sens, au temps de l'explorateur, devaient symboliser la nature dans ce qu'elle pouvait avoir de grandiose, certes, mais aussi d'écrasant pour l'homme: l'arbre (la forêt) et la mer. Or le rapprochement figural de Jolliet avec ces deux objets, loin d'exprimer son écrasement ou sa fragilité, est, au contraire, l'expression d'une force vivant en pleine symbiose avec cette même nature. A cette description, toute mythique, on pourrait juxtaposer, pour seule fin de comparaison, cette autre description de Jolliet, fondée, cette fois, sur une hypothèse ethnique, que l'on retrouve dans la présentation d'une biographie écrite par l'Américaine Virginia S. Eifert:

There is no known protrait of Louis Jolliet. [...] Judging from the general physical attributes of those settling in Quebec in the early days - they were largely from the west of France - some general idea may be gained to throw light on the puzzle. The men of Western France were usually rather stocky, short and wiry, had grey or brown eyes, and dark hair; they were strong men who were well equipped to combat wilderness and Indians in the New World?.

L'hypothèse qu'avance Eifert ne manquant pas de vraisemblance, celleci ne fait que mieux souligner le caractère tout à fait mythique de la description de Jolliet par Grandbois, description, on le voit, issue entièrement de l'imagination du poète, mais en accord avec l'image du personnage que construit le texte dont voici une autre pièce: «lls aimaient Marquette, la Robe-de-la-Douceur. Et son ami, ce jeune chef aux cheveux blonds, bravait les dieux (p. 191)."

A Istoüen, ce jeune Iroquois que Jolliet est sensé avoir retiré des prisons des Keinouchés et qu'il aurait ramené avec lui à Montréal, Grandbois fait tenir les propos suivants, un soir que lui et Jolliet se furent arrêtés sur les bords du lac Huron: "Tu connais Akouessan, qui a longtemps vécu parmi nous, Akouessan, celui que vous nommez Charles Lemoyne? Nous l'aimons tous... Tu... Tu lui ressembles. Tu n'apportes pas le mal... (p. 100).» Le procédé est clair: l'appréciation fortement laudative, fondée sur la comparaison avec une personne dont la réputation est acquise, faite par un membre du parti ennemi, ne peut que créer chez le lecteur un fort mouvement de sympathie envers le personnage dont il est question. Auprès du lecteur, le personnage d'Istoüen fonctionne comme un miroir dans lequel se reflète la figure de Jolliet mais en s'ajustant, toutefois, à une autre figure dont le profil se trouve pour ainsi dire gravé en permanence dans le tain même de la glace. Et au moment de la lecture, c'est ce jeu de réflexion que capte, lui-même surface réfléchissante, l'esprit du lecteur. Par la négative, qui tient autant de la litote que de l'antiphrase: "Tu n'apportes pas le mal...", le discours d'Istouën a pour effet d'attribuer à Jolliet, grâce au pouvoir bénéfique qu'il lui attribue, une capacité de faire le bien à l'exclusion du mal; ce qui le place 
bien au-dessus de l'homme moyen. A rapprocher de la réaction, toute fictive rappelons-le, de ce jeune prisonnier Iroquois, celle que, rapporte le narrateur, eurent les guerriers de la tribu des Maskoutins, membre de la nation du Feu, lorsque leur parla Jolliet durant son voyage de découverte du Mississippi: «Dans un silence attentif, Jolliet exposa les raisons de sa présence et l'objet de sa mission. L'autorité, la franchise de sa parole frappèrent les vieillards. La haute taille impressionna les guerriers (p. 137)." Ici, encore, dans la "fabrication de son personnage", l'auteur emploie le même procédé que dans l'exemple précité avec cette différence que le narrateur intervient directement dans le récit en désignant les vertus appréciées par les indigènes. Toutes sont des vertus " viriles ", marques de l'homme fort, l'homme supérieur selon Grandbois et dont il ne manque pas de multiplier les indices : " La joie durcit certains visages. Une joie forte d'homme fort. Jolliet eut la face dure. II contint sa joie, la réprima (p. 128). " Hemingway ne sut pas mieux s'exprimer! On voit ici comment Grandbois obéit aux stéréotypes culturels. Le laconisme du texte suggère le laconisme du personnage, expression, bien sûr, de la force de caractère. Car un homme fort est un homme au discours parcimonieux (la volubilité est signe de légèreté) et qui doit, en toutes circonstances, maitriser ses sentiments et ses passions dont l'affiche est le propre, de toute évidence, de la femme. L'usage du stéréotype ici est efficace: avec une économie de moyens, il permet la fabrication du héros. D'ailleurs, tout héros légendaire, pour être cela justement, doit nécessairement tenir du stéréotype, c'est-à-dire que la complexité psychologique, qui suppose un mélange de vertus, parfois contradictoires, et de défauts, doit être rigoureusement exclue de sa composition. Plus, ce type de héros doit, jusqu'à en devenir l'exemple même, incarner une ou deux vertus réduites à leur expression la plus simple.

Et c'est ce que fabrique Grandbois dans $N e ́$ à Québec. Sous sa plume, Louis Jolliet devient la vivante image de la rectitude et du courage: «il jugeait, affirme le narrateur, selon sa raison, qu'il avait forte, saine, et dépouillée de bassesse (p. 172). "A la toute fin de son texte, Grandbois, en deux esquisses rapides, achève le portrait de Jolliet: celui-ci, loin de décliner avec l'âge, prend de la force, car le combat contre les éléments de la nature l'a aguerri, fortifié. Tel un dieu, le temps n'a plus aucune prise sur lui:

L'âge ne semblait toucher Jolliet que pour augmenter sa puissance et sa force. Hâlé par le soleil, les vents, la neige, son visage avait pris la teinte du cuir. II était plus grand qu'aucun de ses fils (p. 203).

Le processus de divinisation, auquel Grandbois, tout le long du texte, soumet son personnage, subit ici une très nette accélération (augmentation de sa puissance, taille dépassant celle de ses fils) pour enfin, à la dernière page du texte aboutir à la transformation finale: "Il avait atteint l'âge où la course du sang s'établit sur un rythme définitif, où la vie prend des apparences éternelles (p. 204). " A un âge où la dégradation et la décomposition, auxquelles est soumis normalement le corps de l'homme, soulignant le progrès inéluctable de son entropie, le corps de Louis Jolliet, du moins aux 
mains d'Alain Grandbois, fait preuve au contraire d'une vitalité néguentropique tout a fait étonnante, achevant ainsi sa séparation d'avec l'humanité. Et c'est aussi précisément ce moment-là que choisit Grandbois pour faire " disparaître" (et non mourir) son héros. Ainsi, par touches successives, dans la progression du texte, Grandbois crée un personnage qui, en son développement, se détache de la sèche rigueur historique pour accéder à l'existence du héros mythique.

Cependant, il faut souligner ici que ce passage de l'histoire au mythe s'inscrit dans un effort transformationnel plus large dont le but est de tirer de cette masse documentaire historique un texte ayant forme de récit. Parmi tous les efforts de structuration déployés par Grandbois, il en est un qui mérite une attention particulière: il s'agit de la relation antagonique dans laquelle Grandbois a enfermé les deux grands découvreurs de l'époque: Louis Jolliet et Cavelier de La Salle.

Bien que fondé sur deux rencontres seulement, dans le texte de Grandbois, (dont la première relève de la fiction pure puisqu'elle eut lieu en réalité entre La Salle et Adrien Jolliet, le frère aîné de Louis), cet antagonisme réussit, néanmoins, grâce aux allusions, à innerver tout le texte et fonde ainsi une des structures actantielles qui soutient le récit ${ }^{B}$. Or, cette structure actantielle a ceci de particulier que, fondamentalement, elle n'a pas d'autres fonctions que de faire valoir les qualités morales de Jolliet. Par exemple, dans sa narration des délibérations du “ parlement d'eau-de-vie», Grandbois, par la juxtaposition de deux commentaires, l'un au sujet de Jolliet, l'autre au sujet de La Salle, force en quelque sorte le lecteur à établir une comparaison entre ces deux personnages:

L'occasion s'offrait à lui (Jolliet) de reconquérir une faveur disparue en jouant le jeu du gouverneur. II la méprisa. [...] La Salle, convoqué au dernier moment, s'affirmait partisan convaincu des idées de Frontenac (p. 173).

Rien dans le contexte ne justifie la singularisation de l'attitude de La Salle sinon que cela permet, par voie de contraste, d'accorder plus de relief aux qualités morales de Jolliet. Autrement dit, La Salle n'a, ici comme ailleurs, chaque fois que le texte le mentionne, aucun autre rôle que de doubler, mais négativement, Jolliet. Image spéculaire de celui-ci, mais en creux, le personnage de La Salle, tel qu'il se présente dans le texte de Grandbois, incarne, dirait-on, le manque nécessaire à l'étaiement de la figure hautement vertueuse de Jolliet. Comme le Caïn de la mythologie hébraïque, il est le point de comparaison négatif qui attire et accumule les défauts afin de permettre à l'autre pôle l'épanouissement de la vertu. Personnage travaillé par la sombre et folle passion du mirage de la fortune ( "il avait la taille fière, le teint hâlé, les yeux brûlants (p. 165) "), figure démonique par excellence, le Cavelier de La Salle de Grandbois s'oppose ainsi à la figure solaire de Jolliet, homme d'équilibre et de sérénité. Toutefois, à la fin de son récit, Grandbois, d'une certaine façon, "réhabilite" La Salle en faisant effectuer par Jolliet une volteface à son égard: 
La Salle! (Jolliet se reprochait maintenant de l'avoir haï). L'adversité, comme le feu trempe le fer, avait fait un héros de l'aventurier rouennais (p. 185).

Désormais inutile pour le récit qui s'achève, la figure de La Salle s'ajuste à celle que lui a réservé une certaine histoire: celle de héros et l'antagonisme disparaît en se résorbant dans l'identique. Enfin, il importe de souligner que cet antagonisme repose dans Né à Québec sur une structure antagonique plus large, celle qui opposa Frontenac aux Jésuites, et qui, pour être historiquement avérée, ne semble pas, toutefois, avoir été aussi radicale que Grandbois n'eût voulu le faire croire. Car s'il est vrai qu'il y eut entre les Jésuites et le gouverneur des malentendus, il n'y eut jamais, comme laisse entendre Grandbois, de lutte ouverte entre les deux parties:

All in all, affirme Eccles, Frontenac's relations with the Jesuits can be summed up as frustrated hostility (...) The really amazing thing is that, although Frontenac quarrelled with almost everyone in a position of authority in the colony, he never had any open dispute with the Jesuits?.

Si, en créant cette double opposition, Grandbois réussit à augmenter la tension dramatique de son récit, ce n'est pas, cependant, sans avoir eu, par la même occasion, à sacrifier ici et là, la vérité historique. L'exemple suivant permettra de dépister les gauchissements que Grandbois fait subir au texte historique dans le but justement de creuser les conflits, de les rendre plus profonds, plus efficaces aussi du point de vue romanesque. Le 28 avril 1677, dans une lettre de Colbert adressée à Duchesneau, le successeur de Talon, le ministre fait part de son refus d'accéder à la demande de Jolliet de s'établir dans le pays des Illinois:

Sa Majesté ne veut point accorder au Sieur Jolliet la permission qu'il demande de s'aller établir avec vingt hommes dans le pays des Illinois. II faut multiplier les habitants avant de penser a d'autres terres, et c'est ce que vous devrez avoir pour maxime à l'égard des nouvelles découvertes qui sont faites 10 .

Bien qu'on n'ait pas retrouvé la demande de Jolliet, on peut supposer, d'après la réponse, qu'elle fut adressée à Colbert et ceci par l'entremise de Duchesneau. Or, voici comment les choses se sont passées selon Grandbois:

II vit Frontenac, sollicita une concession chez les Illinois. II s'y établirait avec vingt hommes, élèverait un fort, s'occuperait de culture, de commerce. On repoussa sa demande sous le fallacieux prétexte qu'il ne fallait point éparpiller les forces de la Colonie (p. 169).

On voit ici comment, à la prosaïque rigueur du document historique, Grandbois a préféré l'élaboration d'une manière de petit récit dans lequel s'affrontent les personnages de Frontenac et de Jolliet. Aussi, il me paraît assez remarquable comment Grandbois, par diverses manipulations linguistiques, réussit, sans pour autant la contredire carrément, à infléchir la vérité historique et ce, en jouant uniquement sur le potentiel d'ambiguïté que recèle 
la langue. Dans sa première phrase, lassertion: "ll vit Frontenac..." ne contredit aucun document historique connu mais il va à l'encontre d'une supposition à haute probabilité. Le second segment de cette phrase: "... sollicita une concession chez les lllinois. » pourrait, à la rigueur, être considéré comme ambigu car, et bien que la juxtaposition au segment précédent infère que la demande fut faite à Frontenac, l'absence de lien logique laisse suffisamment de latitude à la phrase pour pouvoir signifier aussi que la demande fut faite auprès d'un autre, peut signifier, en d'autres mots, que les deux actions (voir Frontenac et demander une concession), quoiqu'exécutées par un seul homme, ne visèrent point nécessairement toutes deux Frontenac. De même, le lecteur, à cause du contexte, accorderait au pronom indéfini "On» du début de la dernière phrase, l'antécédent «Frontenac" de la premiere phrase, mais ceci n'est pas la seule lecture possible, car ce pronom pourrait aussi n'avoir aucun antécédent textuel et traduirait ainsi la bureaucratie sans visage ou plutôt aux multiples visages anonymes. En dernier lieu, Grandbois, en employant le qualificatif "fallacieux", non seulement porte un jugement négatif sur les motifs du refus d'accéder à la demande de Jolliet, mais, de plus, suggère fortement que des raisons inavouées et inavouables motivèrent le refus. De ce fait, Frontenac perçu par le lecteur comme un combinard, un maniganceur, se voit discrédité, tandis que Jolliet se voit auréolé de la couronne de la rectitude morale. Ceci dit, que Frontenac fut un trafiqueur d'influence, un homme de duperie, les historiens et les biographes ne laissent aucun doute à ce sujet et il se pourrait bien qu'il fut à l'origine du refus de Colbert, car l'année suivante, celui-ci octroya à Cavelier de La Salle, le protégé du gouverneur, des concessions bien au-delà de ce qu'avait demandé Jolliet pour la même régión.

Cependant, il semble exact qu'il y eut entre Frontenac et Jolliet un refroidissement à partir de 1675 et qui dura jusqu'en 1682. Selon Delanglez ${ }^{11}$, à l'origine de cet antagonisme, l'on retrouva le refus de la part de Jolliet d'épouser les querelles du gouverneur ${ }^{12}$. Et sans doute, pourrait-on prendre ce que certains ont nommé le parlement d'eau-de-vie du 26 octobre 1678 comme exemple de ce qui, essentiellement, séparait ces deux hommes.

Longtemps, l'eau-de-vie fut un sujet de discorde et de dissension en Nouvelle-France. A ceux qui - dont Frontenac - âpre au gain et sans scrupule, voulaient faire fortune rapidement et à peu de frais dans le commerce des fourrures, en défendaient la libre vente aux Indiens, s'opposaient des hommes qui, par respect pour les indigènes, voulaient ou en abolir complètement l'usage ou en restreindre le commerce. Afin de régler, une fois pour toutes, cet épineux problème, le roi obligea Frontenac à convoquer une réunion de vingt notables de la colonie qui auraient à se prononcer. Ce que fit Frontenac. Quinze membres du parlement votèrent en faveur du maintien du commerce libre de l'eau-de-vie; trois membres demandèrent son abolition pure et simple et deux votèrent plutôt pour en restreindre l'usage. Un de ces deux derniers fut Jolliet. Les résultats furent transmis au rol. Mgr Laval, consterné au plus haut point par cette décision, traversa en France pour faire valoir son point de vue. 
Après de multiples tractations dans les coulisses par les défenseurs des deux parties, le conseil du roi finit par proposer un compromis - le commerce limité de l'eau-de-vie - qui fut accepté. Or, ce qu'il faut signaler ici c'est que si la solution adoptée fut, à peu de choses près, celle que proposa Jolliet lors de la réunion, ce ne fut pas à cause de lui qu'elle fut effectivement proposée et acceptée. Cependant, lorsque Grandbois fait le récit de cette réunion, il laisse entendre, alors qu'il n'en fut rien, que Jolliet y joua un rôle déterminant: « Le procès verbal de l'assemblée fut adressé au roi. L'opinion de Jolliet prévalut (p. 173)\%. On le constate, le procédé qu'emploie ici l'auteur est identique à celui que l'on vient d'analyser plus haut. Grandbois, par sa façon de relater les événements, infléchit la vé rité historique afin d'augmenter la stature de Jolliet, de lui octroyer un prestige qu'il ne posséda pas dans la réalité.

A Grandbois, on a fait le reproche, rapporte Jacques Blais, de s'être, dans la première partie de son œuvre, attardé pendant trop longtemps à narrer quantité d'épisodes fort éloignés de son sujet: « Sur les quarante-cinq épisodes, poursuit l'auteur, que comprend cette partie initiale, huit seulement concernent Louis Jolliet lui-même ${ }^{13}$ ». Cette très longue première partie, selon Blais, se justifie:

Car ce que ce patient préambule veut signifier, c'est précisément la trajectoire rectiligne du destin de Jolliet qui ne dérogera pas du but qu'il s'est fixé, malgré les obstacles qui, tournoyant autour de lui, pourraient entraver ses projets. Cette première partie où s'entrecroisent les fils d'intrigues nombreuses, annonce déjà la réussite future de Jolliet qui parvient, au seuil même de sa vie, à s'extirper du piège, tout entier projeté vers l'avenir 14 .

Sans vouloir juger du bien-fondé de cette explication qui repose, me semblet-il, sur une certaine confusion entre le Jolliet “ réel », à jamais disparu, et le Jolliet de Grandbois, personnage " construit ", de fiction, il existe, selon moi, une autre explication, volontiers prosaïque, qui s'appuie sur ce que j'appellerais, faute de mieux, une hypothèse de fabrication.

Ayant choisi de prendre le grand-père maternel comme le point de départ de son récit, Grandbois eût pu, en quelques lignes, tisser la filiation entre celui-ci et son petit-fils. II a opté, au contraire, pour la relation des hauts faits dont sont parsemés les difficiles débuts de la Nouvelle-France. Bien sûr, une telle fresque comme arrière-fond ne peut qu'aider le lecteur à mieux saisir la dimension même de la geste de Jolliet. Cependant, je ne crois pas que ce soit encore là l'explication. Celle-ci est ailleurs. Elle serait plutôt dans la nature même du sujet. Malgré les apparences (les livres consacrés à Jolliet), la connaissance que l'on a de la vie de Jolliet demeure très parcellaire, la documentation étant, en fin de compte, peu fournie. Et Grandbois dans les deuxième et troisième parties de son récit, a exploité tout 
ce qui est su à son sujet, c'est dire qu'en termes de quantité, c'est un peu mince. Trop mince, sans doute, pour faire un livre, d'où la nécessité d'inclure la relation de faits qui ne touche que très indirectement à la vie de Jolliet. Car quel lien y a-t-il vraiment entre la relation de l'exploit de Dollard des Ormeaux au Long-Sault (p. 55-62) et la biographie de Jolliet? Très peu, il me semble, sauf que Grandbois, à l'instar de l'historien Groulx, traite cet épisode sur le mode héroïque de telle sorte qu'à ses yeux le personnage de Dollard des Ormeaux pût préfigurer celui de Louis Jolliet. Ceci est d'autant plus plausible que, on le verra sous peu, le texte de Grandbois présente l'incident de LongSault comme ayant nourri l'imaginaire du jeune Jolliet. Car la relation du massacre de Long-Sault, comme c'est le cas pour d'autres épisodes qui composent cette première partie, est en fait un récit enchâssé: le texte le présente comme une histoire racontée au jeune Jolliet par le père Dablon: "Avidement, Louis buvait les paroles du Jésuite (p. 62)". Vient ensuite le récit, réduit à l'essentiel, du premier voyage effectué par des explorateurs français vers la Baie d'Hudson suivi immédiatement d'un paragraphe sur la vie de Jolliet à 17 ans, paragraphe qui se termine ainsi:

II croyait en sa vocation. II l'appelait de toute son âme *. Mais s'avouait-il que son désir ne tenait qu'aux seules sollicitations d'une vie aventureuse? En n'apportait-il pas plus d'ardeur et de goût aux péripéties d'une relation de voyage qu'aux subtiles et austères dissertations sur la morale et la métaphysique?... Et le voici, les yeux étincelants, écoutant le P. Dablon (p. 63).

Ce procédé d'enchâssement, on le retrouvera une première fois quelque trente pages plus haut où le texte présente Louis Jolliet d'abord comme un enfant qui écoute:

Le charron recevait parfois des amis. C'étaient des hommes rudes, vêtus d'un cuir couleur de tronc d'arbre. II y avait Guillaume Couture, qui avait subi la torture iroquoise; le navigateur Jean Langlois; Jean Nicolet, dont le prestige auprès des tribus indiennes s'étendait du lac Ontario aux côtés arides du golfe; François Bissot, négociant sagace, calculateur; d'autres encore. Ils fumaient des pipes courtes, s'exprimaient lentement. L'attitude menaçante des Iroquois nourrissait d'inquiètes conversations (p. 31).

Suite à ce paragraphe, Grandbois relate la guerre de génocide qu'entreprirent les Iroquois contre les Hurons en 1649. La dernière phrase de ce paragraphe agit donc comme charnière, comme élément de médiation en introduisant le récit que fera l'auteur de la guerre indienne. Afin de maintenir la fiction de la conversation par le biais de laquelle se fait l'enchâssement du récit, Grandbois, quelques paragraphes plus loin, interrompt brievement celui-ci pour $y$ inclure un commentaire sur les auditeurs que sont les enfants:

- Note: Jolliet fut reçu dans les ordres mineurs. 
Avides, les enfants écoutaient. Ils nourrissaient confusément des désirs de bravoure, d'héroïsme. Puis ils voyaient soudain la mutilation de l'homme, ses poings rongés, et ils se serraient les uns contre les autres, frissonnaient... (p. 33).

Ces textes citationnels appellent des commentaires de plusieurs ordres. D'abord, ceux-ci révèlent, il me semble, de la part de l'auteur une volonté très nette d'intégrer cette première partie à son récit principal selon une méthode qui n'a rien d'historique ; le récit enchâssé est un procédé propre à l'œuvre de fiction alors que l'étude historique, sauf exception, favorise la structure processionnelle. Cela ne signifie pas, cependant, que Grandbois ait escamoté complètement le principe de causalité si cher à l'historien. Alors que ce principe est à l'œuvre constamment dans l'élaboration selon la méthode historique, d'une biographie (telle cause, explicite le texte historique, aura produit, chez le sujet biographique, tel trait, etc.) dans le texte grandboisien il agit d'une façon moins directe. Les récits enchâssés dans cette première partie sont présentés comme autant d'histoires ayant nourri l'imaginaire du jeune Jolliet: ces récits de voyage, d'actes de bravoure et d'héroïsme entendus par le jeune Jolliet sur les lèvres d'un Couture ou d'un Dablon non seulement formèrent l'imaginaire de Louis Jolliet - du moins selon Grandbois - mais fournirent les schémas de toutes les actions qu'il entreprendra ultérieurement. A la source, donc, de toutes les activités de Jolliet, une seule cause dominante: la puissance du texte oral, du texte raconté. Et c'est cette puissance, affirme implicitement le texte de Grandbois, qui servira à modeler la réalité, d'elle que procéderont les gestes que posera le découvreur. Mais n'est-ce pas cela justement, cette force de l'imagination que I'on retrouve à la racine même de Né à Québec? N'est-ce pas aussi cette force, nourrie d'histoires lues et entendues qui motive Abancourt, le grandpère de Jolliet, à émigrer vers la Nouvelle-France?

Fasciné, Grandbois a créé des êtres de fascination. Mais la fascination est un autre nom que l'on donne au désir, ou du moins, peut-on dire que la fascination ne saurait s'exercer sans l'existence d'un arrière-plan de désir, que celle-là n'est, en somme, que la manifestation de celui-ci. Et dans ce sens, le texte de Né à Québec est désir fait corps. Plus exactement, il en est la mise en scène, - puisque fondamentalement tout récit est mise en scène (et en jeu) de la pulsion désirante - et les personnages, Jolliet et Abancourt, en sont les figures emblématiques, car plutôt que la reproduction du désir, ils en constituent la représentation. Ce désir qui traverse et travaille le texte de Grandbois de part en part est avant tout, je dirais, un désir de puissance, ou ce que la conscience chrétienne récupère sous le nom de quéte d'absolu.

Cela se manifeste très tôt dans les fantasmes que prête Grandbois au jeune Abancourt mais c'est surtout, et peut-être un peu curieusement, dans les ruptures formelles du texte qu'il est le plus lisible. A Adrien d'Abancourt ne prête-t-il pas le rêve de la toute-puissance conquérante que connurent certains Espagnols : Cortez, Pizarre, Juan Diaz de Solis ? Significative aussi la valeur symbolique - et son expression - que Grandbois accorde aux armes 
de la famille d'Abancourt. « Ne prophétisaient-elles (les armes) pas quelque merveilleux destin: Elles étaient d'argent à un aigle de gueules, becqué et membré d'or, les ailes étendues. Les ailes étendues !... (p. 16) „Par la reprise du dernier syntagme, suivi de points de suspension et d'exclamation, Grandbois donne à son texte une sorte de prolongement au delà des mots et suggère par ce procédé l'esprit qui se perd dans une jonglerie axée ici tout à la fois sur la puissance, le voyage et la conquête des grands espaces. Que Grandbois ait prêté à l'ancêtre des Jolliet cet appétit de pouvoir me paraît assez révélateur quant à la nature de ses propres pulsions et désirs. Et, on le verra, leur présence se manifeste à d'autres niveaux du texte.

Tout au long du récit, le texte connaît une série de modifications soudaines, perceptibles, d'une façon toute particulière dans la phrase, à la fois dans sa longueur et sa complexité et dans l'usage des temps verbaux. Alors que, d'une façon générale, la phrase dans Né à Québec témoigne d'une grande variété, allant de la phrase simple à la phrase complexe construite en étagement de subordonnées et de relatives, le phrases dans ces courts passages obéissent au seul patron de la phrase simple. De plus, du point de vue des verbes, seul s'y trouve à peu près exclusivement le passé simple, tandis qu'ailleurs, le texte jouit d'une certaine diversité. Dans ces brefs passages, la juxtaposition des phrases simples enlève au récit son relief et produit ainsi un effet de distanciation très sensible à la lecture. Or, ces phrases simples, juxtaposées les unes aux autres, liées par aucun lien logique explicite, avec leur concentration de verbes au passé simple, composent des résumés d'événements réduits à l'essentiel avec effacement complet de l'arrière plan 15. Seuls subsistent les éléments formant ce que Weinrich nomme le premier plan du récit, c'est-à-dire le récit réduit à ce qu'on pourrait appeler son ossature:

[...] Et la vie s'organisa au gré du hasard et des saisons. L'aventure prit tour à tour les visages sans gloire du froid, de la fatigue et de la faim. Abancourt dut apprendre une foule de choses que les livres n'enseignent pas. Et les différents métiers qu'il exerça n'eurent pour but que la satisfaction des besoins les plus essentiels.

II se fit, selon les circonstances, trappeur, chasseur, pêcheur, négociant, matelot, soldat, bûcheron. II remonta des rivières, traversa des lacs, s'enfonça au cceur des forêts, traqua le castor, poursuivit l'orignal, pêcha l'anguille, le saumon, le loup-marin. [...] II disparut un jour en plein golfe. Sa femme le pleura, s'éteignit à son tour. II laissait peu de biens, une fille et un surnom; la Caille (p. 20).

C'est ainsi que, résumant les six ans environ passés en Nouvelle-France, Grandbois met un terme à la narration de la vie d'Abancourt. On ne saurait trop souligner, par rapport au texte qui le précède, le caractère antithétique de ce passage et ce, à tous les niveaux. A la prolifération du texte ( 7 pages environ) dans le détail de la description, se substitue le laconisme ( 1 page) parataxique de la narration. Parallèlement, le temps connait une rapide accélération: la longueur du texte étant inversement proportionnelle à la longueur du temps représenté. Outre cela, du point de vue du contenu, la 
première partie, exception faite du récit de la traversée de l'océan, est entièrement consacrée à la relation des rêves grandioses qui animent le jeune Abancourt, tandis que s'opposent à ceux-ci, dans la seconde partie, ses réalisations en Nouvelle-France. De l'enthousiasme passionné du rêve et de l'illusion, le texte passe subitement à la réalité brute, étale, envers laquelle le narrateur, par la voie de l'ironie, prend ses distances. Ce type de style bref, construction parataxique où domine très nettement la forme verbale du passé simple, l'auteur l'emprunte aussi dans son récit chaque fois qu'il fait état, soit d'une naissance, soit d'un mariage ou d'un décès, c'est-à-dire chaque fois qu'il fait état d'une modification dans la vie strictement personnelle d'un des personnages. Or, cela crée plusieurs effets qu'il importe ici de mettre à jour.

Premièrement, l'économie verbale du texte crée chez le lecteur - à cause justement du phénomène de distanciation, selon lequel le narrateur semble s'abstraire du texte - une impression que l'information véhiculée n'a qu'une importance très secondaire, ou plus justement, le lecteur sent que par l'entremise de cette économie, le narrateur veut lui transmettre l'impression que ces événements sont d'importance très secondaire. Donc, grâce à ces ruptures stylistiques qui interviennent dans Né à Québec, une constatation s'impose: ce sont les actes héroïques, tant réels que rêvés, des personnages qui reçoivent, pourrait-on dire, toute l'attention du narrateur dans la mesure où le lecteur sent fortement sa présence dans le texte, tandis qu'envers les actes quotidiens le narrateur prend ses distances. L'extrait suivant permettra par comparaison avec le texte cité ci-dessus, de constater les différences séparant les deux types de texte:

Au coin du feu, Simone filait. Le chant du rouet berçait l'heure. La lueur d'une flamme plus haute avivait sa joue. La laine glissait doucement entre ses doigts minces, blancs. Plus loin, au fond de la pièce, dans l'ombre tiède, le berceau. Un cri s'en élevait partois. Cri d'oiseau, d'ange... Et Adrien s'approchait de la fileuse, parlait. II lui disait, à voix basse, ces peuples étranges et rusés, ces forêts baignées de rivières, ces torrents chavirant les pépites de cuivre, cette vie large, forte pleine de prodiges, cette renommée qui collait au torse des découvreurs comme un pourpoint rouge. II invoquait les fins d'été qui mûrissaient de lourds raisins, les hivers féériques, les fastueux printemps. Ce domaine pouvait contenir trois Europes! (p. 15).

Alors que ce second texte est expression de rêves de grandeur, de toutes les illusions, créatrices d'un monde projeté entièrement vers l'avenir, donc détaché du réel, le premier texte cité, dénudé de toutes fioritures, réduit à l'essentiel, à l'enregistrement du réel pourrait-on dire, exprime ce que l'auteur présente comme la réalité, c'est-à-dire la vérité. Par la rupture stylistique, le texte marque le clivage entre le monde d'une splendeur tout intérieure, création subjective de l'imagination, et le monde de la réalité objective. Et celui-ci paraît d'autant plus banal, plus plat que l'autre fut grandiose.

Le rêve que prête Grandbois à d'Abancourt, marqué au coin de la puissance, de la grandeur et de la renommée, se fonde d'abord, il me semble, 
sur cet antique désir, profondément humain, de retrouver l'état adamique de l'homme où celui-ci, souverain, règnerait sur une nature innocente. Et assez paradoxalement ce qui, de prime abord, peut paraitre au lecteur comme le récit d'un désir d'accomplissement de soi se révèle plutôt comme un besoin inconscient de retrouver l'état archaïque de son moi. Et, d'ailleurs, dans ce que Grandbois présente presque dérisoirement comme la réalité, c'est-à-dire la réalisation antithétique du fantasme, se retrouve justement le récit de la régression de son personnage:

Chaque saison le voyait revenir, un peu plus sauvage, à Québec. Des muscles nouveaux s'étaient noués sur ses membres. II avait la face cuite, basanée. Ses joues s'étaient creusées. II devenait taciturne. Il eut bientôt les gestes lents, précis et rares de l'homme des solitudes (p. 20).

Loin d'accéder au lieu édénique qu'il faisait entrevoir à sa femme, loin d'avoir trouvé cette royauté fantasmée jadis dans son pays natal, loin d'atteindre l'état fortuné des princes, c'est vers un état de plus en plus primitif qu'il s'est acheminé, remontant le cours de l'évolution vers un stade, suggère le narrateur, guère plus élevé que celui de la bête, en tout cas rejoignant le niveau de l'homme des cavernes. Le texte souligne ainsi les dangers qui guettent celui qui prend ses fantasmes pour des réalités "réalisables". On dirait que Grandbois, par l'entremise de son personnage, après avoir donné libre court à sa rêverie, après en avoir montré toutes les séductions, tient ensuite à en révéler le caractère chimérique. L'ironie de Grandbois, en dévoilant l'abîme qui existe entre le monde du fantasme et celui de la réalité, s'exerce, semble-t-il, ici tout à la fois contre son personnage et contre luimême.

Cette première histoire qui inaugure le récit de Né à Québec joue le rôle, dans l'économie générale de l'œuvre, du " récit inversé " tel que proposé par Greimas ${ }^{16}$, et annonce, d'une certaine manière, le second récit, le " récit posé", celui de la vie de Jolliet. Car, grâce à la filiation parentale entre les deux personnages, le second récit ne fait que poursuivre, après l'échec de la première épreuve, la quête instaurée par le héros du premier. C'est dire que sur le plan mythique, Abancourt et Jolliet peuvent être considérés comme formant un seul et même personnage.

Mais à l'encontre d'Abancourt dont la quête se termine assez brusquement et, d'après Grandbois, dans la désillusion, la quête de Jolliet, amorcée par son voyage au Mississippi, se prolongera jusqu'à sa disparition, vraisemblablement dans le Saint-Laurent, quelque vingt-six ans plus tard avec, toujours, cet espoir d'atteindre enfin la “terre promise».

Telle que la présente Grandbois et telle, également, que la présentent les biographes, la vie de Jolliet ne fut jamais autre chose qu'une longue errance dont les péripéties se déroulèrent tour à tour sur les eaux du Mississippi et la Baie d'Hudson, sur la côte du Labrador ainsi que dans les îles d'Anticosti et Mingan. Condamné à l'errance perpétuelle, Jolliet ne continua pas moins sa quête du lieu parfait. Et chaque fois que cet endroit lui sembla 
à la portée de la main, le destin implacablement le lui ravit. Tel fut le cas de cette magnifique contrée qu'il traversa lors de son voyage de découverte du Mississippi en 1673 et dont il fit parvenir une description à Mgr Laval dans une lettre en date du 10 octobre 1674:

Plusieurs de ces nations font du bled trois fois L'année, des citroüilles et des melons Deau, on ne conoist point la neige mais la pluye seulement. ils ne manquent point de fruits, comme prunes, pommes marons, grenades, ananas, mures semblables a celles de france, mais plus douces et plusieurs petits fruits que je conais pas.

Les oiseaux sont Perdrix, becasses, Cailles, Autruches, perroquets, et coqs D'Indes.

Les Bceufs ou Bufles sy voient comme, aux lles, Partout et en quantité, Jen ay veu et compté Jusques 400 ensemble dans une praire, mais lordinaire est den voir trente ou quarante La viande est excelente, IIs sont facilles à tüer, les cerfs, les biches et le chevreüils ne sont que par endroits: Tous ces sauvages, ces fruits ces oiseaux et ces animaux sont dans un pays plus beau que la france II y a des prairies de trois et quatre Lieues, entouréz de forets de mêsme grandeur audela desqueles les prairies Recomencent, de sorte quil y a autant de l'un que de l'autre ${ }^{17}$.

Que Jolliet eût voulu faire la description de l'éden, assurément, il ne se fût pas pris autrement. Rêvant d'y installer une colonie, le découvreur essuya un refus assez catégorique de la part du roi qui, pour des raisons de sécurité, préféra la consolidation de la colonie déjá établie sur les rives du SaintLaurent.

La seconde tentative se solda également par un échec lorsque les Anglais, à l'automne de 1690, commandés par l'amiral Phipps, remontèrent le fleuve dans le but de s'emparer de Québec et brûlèrent en passant les installations de Louis Jolliet sur les îles Anticosti et Mingan, îles qu'il avait reçues en seigneurie dix ans auparavant des mains du roi Louis XIV. N'est-il pas assez singulier que, de toutes les seigneuries disponibles en 1680 , Louis Jolliet se vit octroyer la seigneurie de l'île Anticosti ? Ni l'Histoire ni Grandbois ne précisent si Jolliet en fit la demande ou non. Mais cela importe peu; ce qui me paraît significatif, par contre, c'est qu'il décida de s'y établir afin d'y vivre en permanence. Errant jusqu'aux confins, et au delà, des contrées connues, travaillant, si l'on peut dire, dans les marges de la Nouvelle-France pour une bonne partie de sa vie, c'est encore loin, très loin des agglomérations d'alors qu'il décida - lorsqu'il s'y décida - de s'installer. L'île d'Anticosti exerça-t-elle sur l'imagination de Louis Jolliet la méme fascination qu'elle exerce sur la nôtre aujourd'hui ? Nul ne saurait le dire mais tant par son immensité que par son isolement splendide, elle dut avoir pour cet homme avide d'espace neuf un attrait certain. Pour l'homme, désormais entré dans la mythologie collective, dans la saga de la collectivité et ce, grâce aux efforts, en bonne partie d'Alain Grandbois, que l'île Anticosti fût l'ultime refuge me semble des plus approprié. Qu'il en fut "délogé" par les Anglais, qu'il fut ensuite prêt à tout reprendre, me paraît exemplaire. 
Que Grandbois fût séduit par le personnage historique de Louis Jolliet, il me semble que j'en comprends mieux maintenant les motifs. Ses nombreux voyages de découvertes ou de prospection, son installation dans lîle Anticosti, sont tous des gestes, sinon d'un marginal, à tout le moins d'un être chez qui l'on devine le goût de l'illimité, qui exercèrent sans doute un puissant attrait sur l'esprit de Grandbois, dans la mesure où ce qu'on pourrait appeler le parcours existentiel de Jolliet lui permit d'une façon anticipatoire de faire la lecture de ses propres désirs, de son propre destin. On dirait que cette soif d'espace, de dépassement en quelque sorte, que Grandbois exprimera tant et tant ultérieurement dans sa poésie, il l'exprima d'abord par personne interposée, sa propre soif, peut-être encore ignorée, en tout cas à peine née, se greffant sur celle, très lisible, de Jolliet, le découvreur.

\section{François GALLAYS Université d'Ottawa}

1. Alain Grandbois, Né à Québec, Montréal, Fides, 1962. Toutes les indications paginales entre parenthèses dans le texte renvoient désormais à cette édition.

2. Il m'a semblé intéressant que Grandbois ait choisi de commencer son récit par la présentation du personnage du grand-père maternel de Louis Jolliet alors que de son père, il ne dit mot. On peut trouver à cela plusieurs raisons. Vivant à Paris à l'époque de la rédaction et s'y plaisant, lié culturellement et émotivement à la France, Grandbois eût pu vouloir souligner au tout début de son œuvre, les racines trançaises de Jolliet. Cela me paraît tout à fait naturel. Et s'il a préféré cette figure à celle du père de Louis Jolliet, c'est peut-stre aussi parce que Jean Jolliet, originaire de Dezannes de l'ancien comté de Champagne, charron de métier, ne présenta pas à son esprit une figure apte à réver l'aventure. Tout le contraire, cet Abancourt, homme d'aucun métier et de tous, au nom qui fleure la noblesse. Car, n'est-ce pas là une figure qui permit à Grandbois de mieux se réver une origine à la mesure même de son âme aristocrate? Cependant, il est une autre raison susceptible d'expliquer ce début de récit, raison élémentaire qui tient plutót à l'ordre de fabrication. Grâce à l'évolution de cette figure, Grandbois fait coïncider le début de son récit avec le voyage d'Abancourt vers la Nouvellecoïncider le début de son récit avec la voyage d'Abancourt vers la NouvelleFrance. C'est dire que, voyage, à l'origine de la fondation d'une lignée nouvelle, sa relation fonde aussi le récit de Grandbois. Cette coïncidence inaugurale inscrit d'emblée le texte de Grandbois dans la très ancienne tradition d'un type de récit que j'appellerais le a récit de fondation » et dont Anabase de Saint-JohnPerse est un des meilleurs exemples dans la littérature d'aujourd'hui. D'ailleurs, dans ce permier texte, Grandbois est tout entier tourné vers les origines. Par l'entremise de Jolliet, les siennes, mais aussi celles d'un territoire. Et le titre l'indique assez clairement: il s'agit bien d'une naissance, d'homme il est vrai, mais par le biais de laquelle c'est la naissance/fondation d'un territoire et d'un peuple qui est évoquée.

Enfin il existe une dernière raison que l'on peut invoquer. Par une sorte de mimétisme inconscient, Grandbois aurait peut-être projeté sur le couple historique, Louis Jolliet et son grand-père Abancourt la même filiation qui reliait Alain Grandbois, par-delà son père, à son grand-père aventurier, celui justement qui 
fit fortune en Australie. Faire commencer le récit de voyages de Louis Jolliet par le grand voyage océanique d'Adrien d'Abancourt, c'est à tout le moins suggérer une relation de causalité entre les deux: dans le grand voyage du grand-père se trouve le germe des voyages subséquents du petit-fils.

3. Il n'est pas du tout sû́r qu'Adrien d'Abancourt sût lire. Chose certaine, sa fille Marie, la mère de Louis Jolliet, ne savait pas écrire: la croix fut sa seule signature.

4. Jacques Blais, Présence d'Alain Grandbois, Québec, PUL 1974, p. 44.

5. Philippe Hamon, « Pour un statut sémiologique des personnages », dans Littérature, no 6, mai 1972, p. 109.

6. Qu'ici Alain Grandbois se soit trompé se conçoit aisément. En 1923, on croyait encore que ce fut Louis Jolliet et non son frère aîné Adrien qui fit le voyage avec Péré jusqu'au lac Huron à la recherche de mines de cuivre. Ce n'est qu'en 1945, dans un article publié dans Mid-America que Jean Delanglez, S.J., par une argumentation fondée sur divers documents et reprise dans son livre (Life and Voyages of Louis Jolliet, 1645-1700, Chicago, 1948, traduit en français en 1950: Louis Jolliet, Vie et voyages, (1645-1700), Les Études de l'Institut d'Histoire de l'Amérique française) démontre qu'il fut impossible que Louis fit ce voyage dans l'Ouest en 1669.

7. Virginia S. Eifert, Louis Jolliet Explorer of Rivers, New York, Dodd, Mead and Company, New-York, 1961, p. VIII.

8. De plus, cette petite phrase sert d'introduction à une digression assez longue (4 pages) où sont relatés très succinctement les faits saillants de la vie de l'aventurier La Salle et à laquelle Grandbois n'a pas su résister tant elle dût lui paraître fascinante, car peu d'hommes dépensèrent une telle somme d'énergie ni manifestèrent un entêtement singulièrement aussi passionné dans la poursuite de leurs chimères que cet homme, si bien que son aventure, toujours sur le point d'aboutir mais chutant d'échec en échec, revêt, en fin de compte, les sombres couleurs de la tragédie. Du point de vue de la narration, ces quatres pages constituent un récit enchâssé dans le récit principal.

9. W.J. Eccles, Frontenac The Courtier Governor, Toronto, McClelland and Stewart, 1959, p. 58.

10. Lettre de Colbert à Duchesneau, le 28 avril 1677 citee par Jean Delanglez, Louis Jolliet, Vie et voyages, p. 227.

11. Jean Delanglez, Louis Jolliet, Vie et voyages, p. 228.

12. Il faut souligner ici qu'entre Frontenac et Jolliet il n'en fut pas toujours ainsi, car, contrairement à ce qu'affirme Grandbois pour qui le conflit exista bien avant le voyage de découverte du Mississippi, leurs relations furent trés bonnes. Jolliet au retour de son voyage vers le Mississippi ne fit-il pas sa cour au gouverneur en donnant à cette rivière le nom de Buade et aux régions découvertes celui de Frontenacie.

Que Grandbois fût ici, comme en bien d'autres endroits de son texte, victime de thèses d'historiens, cela est bien possible. En 1895, fut publié le compte de Frontenac par un nommé $H$. Lorin qui soutient, d'une part, que ce fut Talon qui choisit Jolliet et non Frontenac pour aller à la découverte du Mississippi et que, d'autre part, Marquette accompagna Jolliet à l'insu de Frontenac. Or ces deux assertions sont sans aucun fondement dans la réalité. Au retour, Jolliet, dans une lettre adressée au gouverneur écrit qu'il entreprit le voyage « par les premiers ordres que vous (Frontenac) me donnattes, entrant dans votre gouvernement de la Nouvelle-France». Que Talon en fut l'initiateur cela sans doute est-il vrai, mais i) fallait, néanmoins, que Frontenac accorde son autorisation. En 1672 , le supérieur des Jésuites en Nouvelle-France, le père Dablon, dans une lettre à la tête de la Relation de 1671-1672, mentionne le Père et les Français qui furent envoyés à la découverte de la Mer du Sud. II n'eût pas écrit cela, ce me semble, dans une lettre que Frontenac eût sûrement fini par lire, s'il eût fallu lui cacher la présence du "Père». 
13. Jacques Blais, Présence d'Alain Grandbois, p. 48.

14. Op. cit.

15. Voir à ce sujet Harold Weinrich, Le Temps, Paris, Le Seuil, 1973, p. 118.

16. A.J. Greimas, dans Du Sens, Paris, Le Seuil, 1970, p. 187.

17. Jean Delanglez, Louis Jolliet, Vie et voyages, Appendice C, p. 404. 